\title{
Análise dialélica na avaliação do potencial de híbridos de Pinus elliottii x Pinus caribaea para a formação de populações de melhoramento
}

\section{Diallec analysis in assessing the potential of Pinus elliottii x Pinus caribaea hybrids to develop populations for genetic improvement}

\author{
Evandro Vagner Tambarussi' ${ }^{1}$, Emanuelli Granetto Marques², \\ Gisela Maria Pedrassani Andrejow ${ }^{3}$, Fabiana Schmidt Bandeira Peres ${ }^{1}$ e \\ Fernanda Bortolanza Pereira ${ }^{4}$
}

\begin{abstract}
Resumo
Uma das dificuldades no melhoramento florestal é o longo ciclo de seleção de materiais genéticos superiores em qualidade e produtividade, problema que pode ser contornado via seleção precoce de híbridos. $O$ objetivo da presente pesquisa foi estimar os parâmetros genéticos para caracteres silviculturais e verificar a viabilidade da seleção precoce em híbrido de Pinus elliottii x Pinus caribaea, visando a formar uma população base de melhoramento. $O$ experimento foi estabelecido em delineamento em blocos ao acaso, com seis blocos, parcelas lineares de seis plantas e 24 tratamentos (21 progênies de polinização controlada e três testemunhas). Os caracteres diâmetro à altura do peito (DAP) e altura total (H) foram avaliados no quarto e oitavo ano após o plantio. Os quadrados médios referentes às capacidades geral (CGC) e específica (CEC) de combinação foram significativos para a $\mathrm{H}$ aos quatro e oito anos e para DAP aos oito anos. As diferenças significativas entre os tratamentos indicam a existência de variação genética, sugerindo a possibilidade de ganhos com a seleção. As correlações genéticas entre caracteres e entre idades foram altas, variando de 0,96 a 0,99 , indicando que a seleção precoce pode ser uma ferramenta efetiva para implantação de uma população de melhoramento para o híbrido.
\end{abstract}

Palavras-chave: melhoramento genético; correlação genética; seleção precoce; população sintética; SRIPS.

\begin{abstract}
One of the difficulties faced in forest tree improvement programs is the length of the cycle required for genetic selection of superior material in quality and productivity; a problem that can be solved through early selection of hybrids. The objective of the present study was to estimate genetic parameters related to silvicultural traits and verify the feasibility of early selection in Pinus elliottii $x$ Pinus caribaea hybrids aiming to form a breeding population. The experiment was established using a randomized complete block design, with six blocks, linear plots of six plants and 24 treatments $(21$ pollinated progenies and three controls). Diameter at breast height $(\mathrm{DBH})$ and total height $(\mathrm{H})$ were measured at the fourth and eighth year after planting. The mean squares for CGC and CEC were significant for $\mathrm{H}$ at four and eight years of age and for $\mathrm{DBH}$ at eight years of age. Significant differences between treatments indicate the existence of genetic variation and suggest the possibility of gains with selection. Genetic correlations between the characteristics and between the ages were high, ranging from 0.96 to 0.99 , indicating that early selection can be an effective tool for the implementation of a breeding population of the hybrid.
\end{abstract}

Keywords: genetic improvement; genetic correlation; early selection.

\section{INTRODUÇÃO}

O Brasil apresenta um elevado potencial produtivo para florestas plantadas, sendo líder mundial de produtividade florestal. Dentre os dois principais gêneros cultivados no pais, Eucalyptus spp. e

\footnotetext{
1Professor(a) Adjunto(a). UNICENTRO - Universidade Estadual do Centro-Oeste, PR 153, Km 7 - 84500000 - Irati, PR, Brasil. E-mail: tambarussi@gmail.com; fperes@unicentro.br.

${ }^{2}$ Graduanda em Engenharia Florestal. UNICENTRO - Universidade Estadual do Centro-Oeste, PR 153, Km 7 - 84500000 - Irati, PR, Brasil. E-mail: emanuelli_granetto@hotmail.com.

${ }^{3}$ Doutoranda em Ciências Florestais. UFPR - Universidade Federal do Paraná. Rua XV de novembro, 1299, 800600000 Curitiba, PR, Brasil. E-mail: gisela.andrejow@rigesa.com.br.

${ }^{4}$ Doutoranda em Ciências Florestais. Unesp - Universidade Estadual “Júlio de Mesquita Filho / FCA - Faculdade de Ciências Agronômicas. Rodovia Alcides Soares, Km 3 - 18610-307 - Botucatu, SP, Brasil. E-mail: fbp.engflorestal@gmail.com.
}

Sci. For., Piracicaba, v. 46, n. 119, p. 395-403, set. 2018 DOI: dx.doi.org/10.18671/scifor.v46n119.07 
Pinus spp., este último se destaca na Região Sul do país em função das condições edafoclimáticas locais, abastecendo o mercado interno e externo com matéria-prima para as indústrias de papel e celulose baseadas em fibras longas, madeira serrada, painéis compensados e painéis reconstituídos (IBÁ, 2017).

Visando a expansão da base florestal com madeira de Pinus spp., tem-se investido no estudo e introdução de novas espécies deste gênero e no avanço em programas de melhoramento genético. Como exemplo destes esforços na década de 2000 foram implantados talhões experimentais com híbridos interespecíficos de Pinus elliottii var. elliottii Engelm. x Pinus caribaea var. hondurensis (Sénécl.) W.H. Barrett \& Golfari, de alto potencial sob o ponto de vista silvicultural no que diz respeito à produção de madeira (ALMEIDA et al., 2012) e na produção de painéis compensados para emprego na construção civil, fabricação de móveis, embalagens e caixas (ALMEIDA et al., 2014, 2012; SURDI et al., 2015).

Os híbridos interespecíficos têm grande importância nos programas de melhoramento genético quando diferentes caracteres de interesse estão presentes em espécies distintas (ASSIS; MAFIA, 2007). Dentre as estratégias de melhoramento baseadas em hibridação, se destacam a seleção de genitores e o direcionamento dos cruzamentos dentro de programas de Seleção Recorrente Recíproca (SRR) entre populações divergentes e também para a Seleção Recorrente Intrapopulacional em População Sintética (SRIPS). A SRIPS consiste na seleção baseada em população híbrida sintética oriunda do cruzamento entre clones operacionais, com incorporação de novos clones a cada geração (RESENDE; BARBOSA, 2005).

Neste interim, a avaliação genética dos candidatos à seleção é um processo fundamental ao melhoramento genético de plantas, pois os valores genéticos devem ser baseados nos valores genéticos aditivos, ou seja, aqueles que são transmitidos para a descendência (RESENDE, 2002). Para avaliação de cruzamentos híbridos os esquemas de cruzamento em dialelo são frequentemente utilizados em programas de melhoramento genético, tendo sido desenvolvidos vários métodos para análise e interpretação dos mesmos, em função dos objetivos do pesquisador (CRUZ et al., 2012).

Dentre os métodos existentes, o método desenvolvido por Griffing (1956) permite obter informações sobre a capacidade geral (GCG) e específica (CEC) de combinação dos genitores em cruzamentos artificiais. O termo CGC refere-se ao desempenho médio das progênies de um indivíduo quando cruzado com outros indivíduos da população, e a CEC refere-se ao comportamento de um determinado cruzamento em particular, ambas sendo estimativas essenciais para avaliação do valor dos genitores para estabelecimento de populações de hibridação (CRUZ et al., 2012; FALCONER; MACKAY, 1996).

Além disso, para Pinus spp. cujos ciclos de seleção podem variar entre 15 a 25 anos, pode-se buscar empregar a seleção precoce, por permitir identificar características de interesse comercial em árvores na idade juvenil (AGUIAR et al., 2015) possibilitando predizer o desempenho do indivíduo em idade adulta, o que reduz o ciclo de seleção e acelera a obtenção de materiais mais produtivos (ZOBEL; TALBERT, 1984).

Para avaliação da seleção precoce, têm-se estimado as correlações genéticas entre as idades precoces e de rotação, predizendo-se a eficiência de ganhos esperados com a seleção em diferentes idades (FALCONER; MACKAY, 1996; PALUDZYSZYN FILHO et al., 2002), de modo a definir o tempo necessário para aplicar a seleção sem comprometer a eficiência e finalidade do processo (ZOBEL; TALBERT, 1984).

Dessa forma, a presente pesquisa teve como objetivo verificar a viabilidade da seleção precoce de genitores para estabelecimento de populações de hibridação (populações sintéticas) para obtenção de híbridos de P. elliottii x P. caribaea, estimando os parâmetros genéticos para caracteres silviculturais, bem como as capacidades gerais (CGC) e específicas (CEC) de combinação a partir de testes de progênies de polinização controlada.

\section{MATERIAL E MÉTODOS}

\section{Delineamento Experimental}

O teste de progênies de polinização controlada de P. eliiottii x P. caribaea, foi instalado em setembro de 1997, no município de Canoinhas, Estado de Santa Catarina. Segundo dados da empresa 
detentora do material genético em estudo, o clima local caracteriza-se por médias de temperatura anual de $17,9^{\circ} \mathrm{C}$, com mínima absoluta de $-7^{\circ} \mathrm{C}$, precipitação média anual de $1.589,6 \mathrm{~mm}$, sendo classificado como do tipo Cfb da classificação de Köppen.

$\mathrm{O}$ experimento foi delineado em blocos ao acaso, com seis blocos, onde as progênies foram dispostas em parcelas lineares de seis plantas, em espaçamento de 2,5 x 2,5 m. No total, foram estabelecidos 24 tratamentos, sendo 21 progênies de irmãos-completos híbridos e três tratamentos controle compostos de um mix de famílias de polinização aberta de Pinus taeda L.. Os cruzamentos híbridos foram realizados entre dois grupos de genitores: i) Grupo 1 (G1) - seis genitores maternos da espécie P. elliottii; ii) Grupo 2 (G2) - sete genitores paternos de P. caribaea.

As avaliações foram realizadas aos quatro e oito anos de idade, nos anos de 2001 e 2005 respectivamente. As variáveis mensuradas foram altura total da árvore $(\mathrm{H}, \mathrm{m})$ e diâmetro à altura do peito $(\mathrm{DAP}, \mathrm{cm})$.

\section{Obtenção das estimativas dos parâmetros genéticos e capacidades de combinação}

Para obter as estimativas dos componentes das variâncias, das herdabilidades e das correlações fenotípicas, foram utilizados os modelos 147 e 105, implementados no software Selegen REML/ BLUP (RESENDE, 2002), que considera o seguinte modelo estatístico:

$$
\boldsymbol{y}=\boldsymbol{X r}+\boldsymbol{Z g}+\boldsymbol{W p}+\boldsymbol{e},
$$

em que: $y$ é o vetor de dados, $r$ é o vetor dos efeitos de repetição (assumidos como fixos) somados à média geral, $g$ é o vetor dos efeitos genotípicos individuais (assumidos como aleatórios), $p$ é o vetor dos efeitos de parcela, $\boldsymbol{e}$ é o vetor de resíduos (aleatórios). As letras maiúsculas representam as matrizes de incidência para os referidos efeitos (RESENDE, 2007). As correlações genotípicas entre os caracteres e as idades avaliadas foram estimadas por meio do software Selegen REML/BLUP, modelo 105 (RESENDE, 2002).

Os efeitos da capacidade geral de combinação $\left(\hat{g}_{i}\right)$ e da capacidade específica de combinação $\left(\hat{s}_{i j}\right)$ foram estimados por meio das fórmulas:

$$
\hat{\mathrm{g}}_{i(n)}=\bar{x}_{n}-\mu
$$

e

$$
\hat{\mathrm{s}}_{i j(n 1 x n 2)}=v_{o b s}-v_{a n t},
$$

sendo que,

$$
v_{a n t}=\mu+\hat{\mathrm{g}}_{i(n 1)}+\hat{\mathrm{g}}_{i(n 2)}
$$

em que $\bar{x}_{n}$ é a média do genitor $n$, $\mu$ é a média do teste, $v_{o b s}$ é o valor observado e $v_{a n t}$ é o valor antecipado.

\section{Análise de variância}

Os dados foram submetidos à análise de variância (ANOVA) em duas etapas, considerando a estrutura experimental e posteriormente o delineamento genético. Para a primeira parte da análise considerou-se o modelo:

$$
Y_{j k l m}=\mu+b_{j}+c_{k}+t_{l(k)}+e_{j l}+d_{j l m}
$$

em que $Y_{j k l m}$ é a observação fenotípica obtida na planta $m$ do tratamento $l$ no bloco $j, \mu$ é média geral das observações, $b_{j}$ é o efeito do bloco $j$, $c_{k}$ é o efeito do grupo $k$ de tratamentos $(k=1$ para genitores e $k=2$ para cruzamentos), $e_{j l}$ é o erro experimental relativo à parcela $j l$, e $d_{j l m}$ é o erro experimental relativo à planta $m$ do tratamento $l$ no bloco $j$.

Na segunda etapa da ANOVA, para a análise dos cruzamentos dialélicos, foi utilizada a metodologia proposta por Griffing (1956), modelo I, que é específico para dialelos com $n^{2}$ combinações dos $\boldsymbol{n}$ cruzamentos. Tal modelo gera informações a respeito da concentração de genes predominantemente aditivos em seus efeitos (CGC) e dos genes de efeito basicamente não-aditivo (dominância e epistasia) (CEC). Cruz et al. (2012) demonstram os procedimentos para a análise dos dados com essa estrutura, a partir do modelo:

$$
t_{l}=g_{n}+g_{n},+s_{n n}
$$


Neste desdobramento, $g_{n}$ e $g_{n^{\prime}}$ são os efeitos de capacidade geral de combinação dos genitores $n$ e $n^{\prime}$, respectivamente $s_{n n}$, é o efeito da capacidade específica entre os genitores $n$ e $n^{\prime}$. As análises de variância foram efetuadas no ambiente computacional R (R CORE TEAM, 2014).

\section{RESULTADOS E DISCUSSÃO}

\section{Capacidades de combinação, desempenho dos genitores e dos híbridos}

Para a ANOVA com desdobramento de tratamentos em CGC e CEC, os caracteres avaliados apresentaram diferenças significativas entre os tratamentos nas duas idades, indicando a existência de variação genética com predomínio de efeitos genéticos aditivos, dados os maiores quadrados médios observados para as CGC's em relação a CEC (Tabela 1).

Tabela 1. Análise de variância com desdobramento de tratamentos enfocando a capacidade geral (CGC) e específica (CEC) de combinação dos caracteres diâmetro à altura do peito (DAP, $\mathrm{cm})$ e altura $(\mathrm{H}, \mathrm{m})$ aos quatro (DAP4, H4) e aos oito (DAP8, H8) anos de idades em híbridos de Pinus elliottii x Pinus caribaea.

Table 1. Analysis of variance with split treatments focusing on the general (CGC) and specific (CEC) combining ability for diameter at breast height (DAP, cm) and plant height $(H, m)$, at four (DAP4, H4) and eight (DAP8, H8) years of age in Pinus elliottii x Pinus caribaea hybrids.

\begin{tabular}{|c|c|c|c|c|c|c|c|c|c|}
\hline \multirow{2}{*}{ F.V } & \multirow{2}{*}{ G.L } & \multicolumn{2}{|c|}{ DAP4 } & \multicolumn{2}{|c|}{$\mathrm{H} 4$} & \multicolumn{2}{|c|}{ DAP8 } & \multicolumn{2}{|c|}{$\mathrm{H8}$} \\
\hline & & QM & p-valor & QM & p-valor & QM & p-valor & QM & p-valor \\
\hline Repetições & 5 & 31,70 & - & 5,68 & - & 7,75 & - & 4,52 & - \\
\hline Tratamentos & 20 & 21,63 & $<0,05$ & 6,66 & $<0,05$ & 45,72 & $<0,05$ & 21,86 & $<0,05$ \\
\hline CGC (G1) & 6 & 26,35 & 0,12 & 7,39 & $<0,05$ & 61,98 & $<0,05$ & 18,08 & $<0,05$ \\
\hline CGC (G2) & 4 & 22,21 & 0,18 & 8,54 & $<0,05$ & 54,07 & $<0,05$ & 46,12 & $<0,05$ \\
\hline CEC & 10 & 18,57 & 0,20 & 5,46 & $<0,05$ & 32,63 & 0,11 & 14,42 & $<0,05$ \\
\hline Erro Entre & 23 & 12,87 & - & 1,91 & - & 18,74 & - & 5,32 & - \\
\hline Erro Dentro & 615 & 4,99 & & 1,05 & & 14,51 & & 3,44 & \\
\hline$\hat{C} V \%$ & & 32,80 & & 24,30 & & 21,50 & & 17,60 & \\
\hline
\end{tabular}

FV, Fonte de variação; GL, Graus de liberdade; QM, Quadrados médios; CGC (GI), Capacidade geral de combinação do genitor feminino; CGC (G2), capacidade geral de combinação do genitor masculino; $C E C$, capacidade específica de combinação, $\hat{C} V$ \% coeficiente de variação experimental.

Resultados semelhantes aos observados para CGC's e CEC foram encontrados por Paula et al. (2002) e Santos et al. (2006) ao avaliarem caracteres de crescimento em diferentes espécies florestais, o que evidencia que os materiais genéticos usados no setor florestal apresentam, de modo geral, grande variação genética, garantindo ganhos a longo prazo nos programas de melhoramento (MASSARO et al., 2010).

Considerando apenas a CGC, em ambos os grupos de genitores (G1 e G2) os efeitos foram não significativos para o DAP aos quatro anos de idade (Tabela 1). Tomando as CGC's significativas para DAP aos oito anos associada à ausência de significância na estimativa da CEC, infere-se que no controle genético desse caractere há predominância de efeitos aditivos. Assim, para DAP, o desempenho dos cruzamentos pode ser previsto com base apenas nas estimativas da CGC, (GOMIDE et al., 2008) de forma que as populações sintéticas mais promissoras serão aquelas originadas de cruzamentos que envolvem dois genitores com alta CGC e pertencentes a grupos distintos.

Os efeitos da CEC foram significativos somente para $\mathrm{H}$ nas duas idades avaliadas (Tabela 1), evidenciando a existência de efeitos genéticos aditivos e não aditivos no controle do mesmo, resultado similar ao observado por Teixeira et al. (2013) em clones de Eucalyptus grandis W. Hill x Eucalyptus urophylla S.T. Blake. Isto indica que o desempenho de híbridos para H depende tanto da CGC como da CEC dos genitores.

Em relação a estimativa do efeito da CGC $\left(\hat{g}_{i}\right)$ para cada genitor, altas estimativas positivas ou negativas, referem-se à superioridade ou inferioridade do mesmo em relação aos demais genitores avaliados (CRUZ et al., 2012). Baseados nos valores de $\hat{g}_{i}$ (Tabela 2), os melhores genitores maternos foram 01, 02 e 06, enquanto os melhores genitores paternos foram 11, 08 e 07.

Uma vez que o efeito da CEC $\left(\hat{s}_{i j}\right)$ pode ser interpretado como o desvio do híbrido em relação ao que seria esperado com base na CGC dos genitores, valores absolutos elevados de $\hat{s}_{i j}$ demonstram que o cruzamento específico foi melhor (valor positivo) ou pior (valor negativo) do que o esperado com base na CGC dos genitores (CRUZ et al., 2012). Para DAP, os cruzamentos que tiveram um desempenho superior ao esperado em ambas as idades foram 01x07, 04x13 e 03x11, enquanto para $\mathrm{H}$, os cruzamentos que se destacaram foram 04x08 e 05x10 (Tabela 3). 
Tabela 2. Estimativa dos efeitos da capacidade geral de combinação $\left(\hat{g}_{i}\right)$ para os caracteres diâmetro à altura do peito (DAP, cm) e altura $(\mathrm{H}, \mathrm{m})$, aos quatro (DAP4, H4) e oito (DAP8, H8) anos de idade em híbridos de Pinus elliottii $x$ Pinus caribaea.

Table 2. Estimated effects of general combining ability $\left(\hat{g}_{i}\right)$ for diameter at breast height (DAP, $\left.\mathrm{cm}\right)$ and plant height $(\mathrm{H}, \mathrm{m})$, at four (DAP4, H4) and eight (DAP8, H8) years of age in Pinus elliottii x Pinus caribaea hybrids.

\begin{tabular}{|c|c|c|c|c|c|}
\hline \multirow{2}{*}{ Genitores } & & \multicolumn{4}{|c|}{$\hat{\boldsymbol{g}}_{i}$} \\
\hline & & DAP4 & $\mathrm{H} 4$ & DAP8 & $\mathrm{H8}$ \\
\hline \multirow{6}{*}{ G1 (P. elliottii) } & 1 & 1,29 & 0,45 & 2,22 & 0,36 \\
\hline & 2 & 0,68 & 0,34 & 0,70 & 0,64 \\
\hline & 3 & $-0,38$ & 0,08 & $-0,53$ & $-0,01$ \\
\hline & 4 & $-0,36$ & $-0,05$ & $-0,53$ & $-0,44$ \\
\hline & 5 & 0,17 & $-0,20$ & 0,38 & $-0,07$ \\
\hline & 6 & 0,48 & 0,33 & 0,87 & 0,88 \\
\hline \multirow{7}{*}{ G2 ( $P$. caribaea) } & 7 & 1,29 & 0,45 & 2,22 & 0,36 \\
\hline & 8 & 0,95 & 0,36 & 1,54 & 0,44 \\
\hline & 9 & 0,08 & $-0,06$ & $-0,15$ & 0,14 \\
\hline & 10 & $-0,39$ & $-0,23$ & $-0,39$ & $-0,49$ \\
\hline & 11 & 0,21 & 0,43 & 0,58 & 0,80 \\
\hline & 12 & 0,02 & $-0,07$ & $-0,36$ & $-0,22$ \\
\hline & 13 & $-0,33$ & $-0,11$ & 0,06 & 0,10 \\
\hline
\end{tabular}

Tabela 3. Médias gerais de tratamentos e estimativa dos efeitos da capacidade específica de combinação $\left(\hat{\mathrm{S}}_{i \mathrm{ij}}\right)$ para os caracteres diâmetro à altura do peito $(\mathrm{DAP}, \mathrm{cm})$ e altura $(\mathrm{H}, \mathrm{m})$, aos quatro (DAP4, H4) e oito (DAP8, H8) anos de idade em híbridos de Pinus elliottii x Pinus caribaea.

Table 3. Overall means of treatments and estimated effects of specific combining ability $\left(\hat{\mathrm{S}}_{\mathrm{ij}}\right)$ for diameter at breast height (DAP, cm) and plant height $(\mathrm{H}, \mathrm{m})$, at four (DAP4, H4) and eight (DAP8, H8) years of age in Pinus elliottii x Pinus caribaea hybrids.

\begin{tabular}{|c|c|c|c|c|c|c|c|c|}
\hline \multirow{2}{*}{ Cruzamento } & \multicolumn{4}{|c|}{ MÉDIAS } & \multicolumn{4}{|c|}{$\hat{\mathbf{s}}_{i j}$} \\
\hline & DAP4 & H4 & DAP8 & $\mathrm{H8}$ & DAP4 & $\mathrm{H} 4$ & DAP8 & H8 \\
\hline $1 \times 07$ & 12,22 & 6,13 & 22,38 & 13,46 & 1,29 & $-0,45$ & 2,22 & $-0,36$ \\
\hline $04 \times 13$ & 11,10 & 5,98 & 21,17 & 14,11 & 0,86 & 0,46 & 0,79 & 0,26 \\
\hline $06 \times 10$ & 11,82 & 5,93 & 22,08 & 14,07 & 0,80 & 0,15 & 0,39 & 0,33 \\
\hline $03 \times 11$ & 11,45 & 6,35 & 21,52 & 13,97 & 0,69 & 0,16 & 0,96 & 0,22 \\
\hline $04 \times 08$ & 12,07 & 6,30 & 20,97 & 13,21 & 0,55 & 0,31 & 0,91 & 0,97 \\
\hline $05 \times 10$ & 11,11 & 5,72 & 20,47 & 12,88 & 0,40 & 0,47 & 0,82 & 0,67 \\
\hline $06 \times 12$ & 11,79 & 6,02 & 21,03 & 13,82 & 0,36 & 0,08 & 0,85 & 0,21 \\
\hline $06 \times 11$ & 11,86 & 6,49 & 21,76 & 14,66 & 0,24 & 0,05 & 0,15 & $-0,12$ \\
\hline $05 \times 09$ & 11,34 & 5,33 & 20,86 & 13,44 & 0,16 & $-0,09$ & $-0,26$ & $-0,23$ \\
\hline $04 \times 09$ & 10,58 & 5,58 & 19,48 & 12,71 & $-0,07$ & 0,01 & $-0,01$ & $-0,08$ \\
\hline $05 \times 11$ & 11,23 & 5,86 & 20,13 & 12,94 & $-0,08$ & $-0,05$ & $-0,43$ & $-0,07$ \\
\hline $02 \times 09$ & 11,61 & 6,02 & 20,69 & 13,76 & $-0,08$ & 0,06 & 0,15 & $-0,14$ \\
\hline $06 \times 08$ & 12,24 & 6,58 & 22,14 & 14,17 & $-0,12$ & 0,21 & $-0,43$ & $-0,25$ \\
\hline $05 \times 13$ & 10,47 & 5,24 & 18,79 & 12,08 & $-0,30$ & $-0,13$ & $-0,53$ & $-0,25$ \\
\hline $04 \times 12$ & 10,10 & 5,20 & 20,04 & 12,88 & $-0,49$ & $-0,36$ & $-1,19$ & $-0,65$ \\
\hline $03 \times 10$ & 9,66 & 5,16 & 19,35 & 13,06 & $-0,50$ & $-0,36$ & $-0,45$ & $-0,52$ \\
\hline $04 \times 10$ & 9,57 & 4,98 & 18,31 & 11,32 & $-0,61$ & $-0,41$ & $-0,94$ & $-0,85$ \\
\hline $05 \times 08$ & 11,33 & 5,24 & 20,15 & 13,71 & $-0,72$ & $-0,60$ & $-1,21$ & $-1,09$ \\
\hline $04 \times 11$ & 10,02 & 5,72 & 18,09 & 11,79 & $-0,76$ & $-0,34$ & $-0,86$ & $-0,40$ \\
\hline $06 \times 13$ & 10,24 & 5,50 & 20,87 & 12,38 & $-0,84$ & $-0,40$ & $-0,94$ & $-0,37$ \\
\hline $06 \times 09$ & 10,52 & 5,56 & 19,59 & 13,55 & $-0,97$ & $-0,39$ & $-1,29$ & $-0,57$ \\
\hline Média geral & 10,45 & 5,36 & 18,98 & 12,42 & - & - & - & - \\
\hline
\end{tabular}

De modo geral, o desempenho médio dos híbridos aos quatro e oito anos foi superior ao desempenho médio das testemunhas entre 8,87 e 11,76\% (Tabela 4). Uma vez que um dos objetivos de um programa de melhoramento é melhorar a média da população ao longo do tempo, por meio da introdução e testes de novos materiais (SANTOS et al., 2015), o híbrido em referência, P. elliottii x $P$. caribaea se mostrou um bom candidato para compor o quadro de materiais genéticos operacionais. 
Tabela 4. Média dos híbridos e testemunhas para os caracteres diâmetro à altura do peito (DAP, $\mathrm{cm})$ e altura $(\mathrm{H}, \mathrm{m})$ aos quatro (DAP4, H4) e oito (DAP8, H8) anos de idade em híbridos de Pinus elliottii x Pinus caribaea.

Table 4. Means of hybrids and controls for the characters diameter at breast height $(D A P, c m)$ and height $(H, m)$ at four (DAP4, H4) and eight (DAP8, H8) years of age in hybrids of Pinus elliottii x Pinus caribaea.

\begin{tabular}{lcccc}
\hline & DAP4 & DAP8 & H4 & H8 \\
\hline Média dos híbridos & 10,93 & 20,16 & 5,68 & 13,10 \\
Média das testemunhas & 9,96 & 17,79 & 5,04 & 11,73 \\
Diferença em \% & 8,87 & 11,76 & 11,27 & 10,46 \\
\hline
\end{tabular}

\section{Estimativas dos parâmetros genéticos}

A variância genotípica entre tratamentos $\left(\hat{\sigma}_{g}^{2}\right)$ para o caractere DAP, variou de $2,26 \%$ aos quatro anos para 7,56\% aos oito anos de idade, representando 13,58\% e 12,76\% da variância fenotípica total $\left(\hat{\sigma}_{f}^{2}\right)$, respectivamente. Para altura, os valores variaram de $0,70 \%$ aos quatro anos para 3,57\% aos oito anos, representando $16,62 \%$ e $15,73 \%$ de $\hat{\sigma}_{f}^{2}$, respectivamente (Tabela 5).

Tabela 5. Estimativa das variâncias genéticas (parcela, ambiental e fenotípica) para os caracteres diâmetro à altura do peito (DAP, $\mathrm{cm})$ e altura $(\mathrm{H}, \mathrm{m})$ aos quatro (DAP4, H4) e aos oito (DAP8, H8) anos de idade em híbridos de Pinus elliottii x Pinus caribaea.

Table 5. Estimates of genetic variance (plot, environment, and phenotype) for diameter at breast height (DAP, $\mathrm{cm}$ ) and height $(\mathrm{H}, \mathrm{m})$ at four (DAP4, H4) and at eight (DAP8, H8) years of age in Pinus elliottii $x$ Pinus caribaea hybrids.

\begin{tabular}{lcccc}
\hline \multirow{2}{*}{ Parâmetros } & \multicolumn{5}{c}{ Caracteres } \\
\cline { 2 - 5 } & DAP4 & DAP8 & H4 & H8 \\
\hline$\hat{\sigma}^{2}{ }_{g}$ & 2,26 & 7,56 & 0,70 & 3,57 \\
$\hat{\sigma}^{2}{ }_{\text {parc }}$ & 0,40 & 0,25 & 0,08 & 0,36 \\
$\hat{\sigma}^{2}{ }^{2}$ & 13,98 & 51,44 & 3,43 & 18,77 \\
$\hat{\sigma}^{2}{ }_{f}$ & 16,64 & 59,24 & 4,21 & 22,69 \\
\hline
\end{tabular}

$\hat{\sigma}_{g}^{2}$, Variância genotípica; $\hat{\sigma}_{\text {parc }}^{2}$, Variância ambiental entre parcelas; $\hat{\sigma}_{\mathrm{d}}^{2}$, Variância residual dentro da parcela; $\hat{\sigma}_{\mathrm{f}}^{2}$, Variância fenotípica.

Para ambos os caracteres, as estimativas de $\hat{\sigma}_{g}^{2}$ e $\hat{\sigma}_{f}^{2}$ mostraram nítida tendência de aumento com o avanço da idade, o que já foi relatado em outros trabalhos com espécies do gênero, como o desenvolvido por Xiang et al. (2003) com P. taeda.

As estimativas da herdabilidade individual no sentido restrito $\left(\hat{h}_{a}^{2}\right)$ apresentaram valores muito próximos entre idades para ambos os caracteres avaliados, uma estabilidade que indica que mesmo com o avanço da idade, as variâncias genotípicas influenciam numa mesma magnitude as variâncias fenotípicas dos caracteres (Tabela 6).

Tabela 6. Estimativa das herdabilidades para os caracteres diâmetro à altura do peito $(\mathrm{DAP}, \mathrm{cm})$ e altura $(\mathrm{H}, \mathrm{m})$ aos quatro (DAP4, H4) e aos oito (DAP8, H8) anos de idade em híbridos de Pinus elliottii x Pinus caribaea.

Table 6. Estimation of heritability for diameter at breast height (DAP, $\mathrm{cm}$ ) and height $(\mathrm{H}, \mathrm{m})$ at four (DAP4, H4) and at eight (DAP8, H8) years of age in Pinus elliottii $x$ Pinus caribaea hybrids.

\begin{tabular}{lcccc}
\hline \multirow{2}{*}{ Parâmetros } & \multicolumn{4}{c}{ Caracteres } \\
\cline { 2 - 5 } & DAP4 & DAP8 & H4 & H8 \\
\hline$\hat{h}^{2}{ }_{a}$ & $0,27 \pm 0,07$ & $0,25 \pm 0,07$ & $0,33 \pm 0,84$ & $0,31 \pm 0,08$ \\
$\hat{c}^{2}{ }_{\text {parc }}$ & 0,02 & 0,00 & 0,01 & 0,01 \\
$\hat{h}^{2}{ }_{m p}$ & 0,83 & 0,83 & 0,86 & 0,85 \\
$\hat{A} c_{\text {prog }}$ & 0,91 & 0,91 & 0,93 & 0,92 \\
$\hat{h}^{2}{ }_{\text {ad }}$ & 0,16 & 0,14 & 0,20 & 0,19 \\
\hline
\end{tabular}

$\hat{h}_{a}{ }_{a}$, herdabilidade individual no sentido restrito; $\hat{c}^{2}{ }_{\text {parc }}$, coeficiente de determinação dos efeitos de parcela; $\hat{h}_{m p}^{2}$, herdabilidade da média de progênies; $\hat{A} c_{\text {prog }}$, acurácia da seleção de progênies; $\hat{h}_{a d}^{2}$, herdabilidade aditiva dentro de parcela.

As herdabilidades em nível de média de progênies ( $\hat{h}_{m p}^{2}$ ) variaram muito pouco entre os caracteres e entre idades (Tabela 6). A classificação proposta por Resende (1995) considera herdabilidades de 0,01 a 0,15 como baixas; de 0,15 a 0,50 medianas; e acima de 0,50 altas; de forma que as estimativas de $\hat{h}_{m p}^{2}$ e $\hat{h}_{a}^{2}$ (nível individual) foram consideradas altas e medianas, respectivamente, sugerindo, portanto, baixa influência do ambiente na expressão dos caracteres.

Tambarussi et al. (2010), estudando um teste de progênie de P. caribaea var hondurensis encontraram $\hat{h}_{a}^{2}$ que variaram de 0,28 para DAP a 0,44 para altura, valores estes próximos aos observados na presente pesquisa. Em vários testes de progênies de espécies de Pinus, têm sido estimados $\hat{h}_{m p}^{2}$ para 
os caracteres de crescimento, como os relatados em Pinus caribaea var. bahamensis (Griseb.) W.H. Barrett \& Golfari aos 25 anos (SEBBENN et al., 2008) e P. caribaea var hondurensis aos 14 anos de idade (TAMBARUSSI et al., 2010), cujos valores para $h_{m p}^{2}$ foram inferiores aos encontrados na presente pesquisa para o caractere DAP.

Para ambos os caracteres avaliados em ambas as idades, percebe-se que as acurácias $\left(\hat{A} c_{\text {prog }}\right)$ alcançaram altas magnitudes, variando entre 0,91 e 0,93 (Tabela 6). A acurácia é um parâmetro estatístico muito importante, principalmente nos ensaios finais em programas de melhoramento genético, por inferir sobre a precisão e a qualidade dos experimentos, tendo a propriedade de informar sobre o ordenamento dos genótipos para fins de seleção (RESENDE, 2002).

Os resultados indicam, portanto, que para este material genético, a seleção baseada em médias de progênies tem um nível de precisão maior do que a baseada em plantas individuais, visto que os parâmetros em nível de progênies ( $\hat{A} c_{\text {prog }}$ e $\hat{h}_{m p}^{2}$ ) apresentaram valores superiores, obtendo-se maiores ganhos genéticos se no método de seleção aplicado for considerada somente a divergência genética entre progênies. Além disso, a influência dos erros experimentais torna-se menor quando as análises são baseadas em médias, ao invés de valores individuais (VENCOVSKY; BARRIGA, 1992).

\section{Correlações entre caracteres, viabilidade de seleção precoce e seleção dos genitores}

A correlação fenotípica $\left(\hat{r}_{f}\right)$ pode ser diretamente mensurada a partir de dois caracteres, tendo causas genéticas e ambientais. Entretanto, apenas a correlação genética $\left(\hat{r}_{g}\right)$ envolve uma associação de natureza herdável, uma vez que mede o grau de associação genética entre dois caracteres quantitativos em uma determinada população podendo assim, por consequência, ser utilizada em programas de melhoramento (FALCONER; MACKAY, 1996).

Os valores de $\hat{r}_{f}$ nas combinações estudadas foram positivas, variando de 0,73 a 0,99 , enquanto que os valores de $\hat{r}_{g}$ entre caracteres e entre idades foram altas, positivas e com menor variação $(0,97$ a 0,99$)$ (Tabela 7$)$.

Tabela 7. Estimativas de correlações genotípicas $\left(\hat{r}_{g}\right)$ e fenotípicas $\left(\hat{r}_{f}\right)$ entre os caracteres diâmetro à altura do peito $(\mathrm{DAP}, \mathrm{cm})$ e altura $(\mathrm{H}, \mathrm{m})$ aos quatro (DAP4, H4) e aos oito (DAP8, H8) anos de idade em híbridos de Pinus elliottii x Pinus caribaea ( $p$-valor $<0,05)$.

Table 7. Estimates of genotypic $\left(r_{g}\right)$ and phenotypic $\left(r_{f}\right)$ correlations between diameter at breast height (DAP, $\mathrm{cm})$ and height $(\mathrm{H}, \mathrm{m})$ at four (DAP4, H4) and at eight (DAP8, H8) years of age in Pinus elliottii $x$ Pinus caribaea hybrids (value- $p<0,05$ ).

\begin{tabular}{|c|c|c|c|c|}
\hline \multirow[t]{2}{*}{ Caracteres } & \multicolumn{4}{|c|}{$\hat{r}_{g}$} \\
\hline & DAP4 & DAP8 & $\mathrm{H} 4$ & H8 \\
\hline DAP4 & 1,00 & 0,99 & 0,96 & 0,96 \\
\hline DAP8 & & 1,00 & 0,96 & 0,97 \\
\hline $\mathrm{H} 4$ & & & 1,00 & 0,97 \\
\hline $\mathrm{H} 8$ & & & & 1,00 \\
\hline & & & & \\
\hline Caracteres & DAP4 & DAP8 & H4 & H8 \\
\hline DAP4 & 1,00 & 0,88 & 0,84 & 0,83 \\
\hline DAP8 & & 1,00 & 0,91 & 0,73 \\
\hline $\mathrm{H} 4$ & & & 1,00 & 0,99 \\
\hline $\mathrm{H} 8$ & & & & 1,00 \\
\hline
\end{tabular}

As correlações genéticas indicam que se a seleção for feita com base no DAP aos quatro anos de idade, haverá efeitos positivos de seleção tanto nesta variável quanto na altura das árvores aos oito anos de idade, dados os valores de $\hat{r}_{g}$ iguais a 0,99 e 0,96, respectivamente (Tabela 7). Altas e positivas correlações fenotípicas e genéticas foram encontradas em P. caribaea var hondurensis aos 14 anos de idade (TAMBARUSSI et al., 2010), em Pinus radiata D. Don aos 12, 24 e 36 meses (DIAS et al., 2016) e em Pinus pinaster Aiton aos 17 anos (GASPAR et al., 2008).

Esses valores elevados de correlação genética entre as idades indicam que a seleção precoce poderá ser efetiva. Com o ganho de tempo, fator importante dado o longo período de gerações do melhoramento de espécies do gênero Pinus, a seleção dos genótipos desejados será possível já nas primeiras etapas do programa de melhoramento, reduzindo o ciclo e, consequentemente, permitindo a identificação de genótipos puros ou híbridos mais produtivos, antecipando a oferta de sementes melhoradas (PALUDZYSZYN FILHO et al., 2002) e, no caso do presente estudo, viabilizando a 
Tambarussi et al. - Análise dialélica na avaliação do potencial de híbridos de

Pinus elliottii x Pinus caribaea para a formação de populações de melhoramento

implantação de uma população base sintética para obtenção de híbridos produtivos. Acrescido a isto, as altas e positivas correlações genéticas entre caracteres possibilita a seleção indireta, dado que a seleção direta para um caractere beneficiará o outro (FALCONER; MACKAY, 1996).

A eficiência da seleção em idade precoce em Pinus spp. também foi confirmada em outras espécies do gênero para caracteres silviculturais (PALUDZYSZYN FILHO et al., 2002; DIAS et al., 2016; COUTINHO et al., 2017). Entretanto há que se considerar que a eficiência da seleção precoce é garantida quando a seleção do material é feita em locais com condições edafoclimáticas semelhantes às dos locais onde os genótipos serão cultivados (COUTINHO et al., 2017).

\section{CONCLUSÕES}

O teste de progênies de P. elliottii x P. caribaea apresentou altos níveis de variação genética para os caracteres DAP e $\mathrm{H}$ aos quatro e oito anos de idade, indicando a possibilidade de ganhos com seleção de materiais genéticos.

A superioridade do desempenho médio dos híbridos indica o potencial de uso dos híbridos de P. elliottii x P. caribaea em plantios comerciais.

As altas e positivas correlações genéticas entre as idades indicam que a seleção precoce aos quatro anos de genitores para formação de população sintética pode ser efetiva.

\section{REFERÊNCIAS BIBLIOGRÁFICAS}

AGUIAR, A. V.; SOUSA, V. A.; MORAES, M. L. T.; FREITAS, M. L. M. Ferramentas aplicadas no melhoramento genético de pinus para otimizar o ganho esperado. Série Técnica IPEF, Piracicaba, v. 19, n. 40, p. 40, 2015.

ALMEIDA, N. F.; BORTOLETTO JÚNIOR, G.; MENDES, R. F.; SURDI, P. G. Produção e avaliação da qualidade de lâminas de madeira de um híbrido de Pinus elliottii var. elliottii x Pinus caribaea var. hondurensis. Floresta e Ambiente, Seropédica, v. 21, n. 2, p. 261-268, 2014.

ALMEIDA, N. F.; BORTOLETTO JÚNIOR, G.; MENDES, R. F; SURDI, P. G. Avaliação da madeira de Pinus elliottii var. elliottii x Pinus caribaea var. hondurensis para a produção de compensados. Scientia Forestalis, Piracicaba, v. 40, n. 96, p. 435-443, 2012.

ASSIS, T. F.; MAFIA, R. G. Hibridação e Clonagem. In: BORÉM, A. Biotecnologia Florestal, Viçosa: Ed. UFV, 2007, pp. 93-121.

COUTINHO, R. T.; BESPALHOK FILHO, J. C.; FRISTCHE NETO, R.; FRIZZO, C. Viabilidade da seleção precoce de Pinus taeda L. em diâmetro a altura do peito em programa de melhoramento genético. Scientia Forestalis, Piracicaba, v. 45, n. 113, p. 205-219, 2017.

CRUZ, C. D.; REGAZZI, A. J.; CARNEIRO, P. C. S. Modelos Biométricos Aplicados ao Melhoramento Genético. 3 ed. Viçosa: UFV, 2012, 480 p.

DIAS, P. C.; XAVIER, A.; RESENDE, M. D. V.; BIENARSKI, F. A.; ESTOPA, R. A.; PIRES, I. E. Juvenile-mature genetic correlations in Pinus taeda clones propagated via somatic embryogenesis. Revista Árvore, Viçosa, v. 40, n. 2, p. 255-267, 2016.

FALCONER, D. S.; MACKAY, T. F. Introduction to quantitative genetics. 4 ed. Londres: Longman Group, 1996. $464 \mathrm{p}$.

GASPAR, J. M.; LOUZADA, J. L.; AGUIAR, A.; ALMEIDA, M. H. Genetic correlations between wood quality traits of Pinus pinaster Ait.. Annals of Forest Science, v. 65, n. 7, p.1, 2008

GOMIDE, M. L.; MALUF, W. R.; GOMES, L. A. A. Capacidade de combinação de linhagens elite de pimentão (Capsicum annuum L.). Ciência e Agrotecnologia, Lavras, v.32, p.740-748, 2008.

GRIFFING, B. Concept of general and specific combining ability in relation to diallel crossing systems. Australian Journal of Biological Sciences, Melbourn, v. 9, p. 463-493, 1956. 
IBÁ - INDÚSTRIA BRASILEIRA DE ÁRVORES. Relatório 2017: ano base 2016. Brasília: IBÁ, 2017. 50 p.

MASSARO, R. A. M.; BONINE, C. A. V.; SCARPINATI, E. A.; PAULA, R. C. Viabilidade de aplicação da seleção precoce em testes clonais de Eucalyptus spp. Ciência Florestal, Santa Maria, v. 20, n.4, p. 597-609, 2010.

PALUDZYSZYN FILHO, E.; FERNANDES, J. S. C.; RESENDE, M. D. V. Avaliação e seleção precoce para crescimento de Pinus taeda. Pesquisa Agropecuária Brasileira, Brasilia, v. 37, n. 12, p.1719-1726, 2002.

PAULA, R. C.; PIRES, I. E.; BORGES, R. C. G.; CRUZ, C. D. Predição de ganhos genéticos em melhoramento florestal. Pesquisa Agropecuária Brasileira, Brasília, v. 37, n. 2, p. 159-165, 2002.

R CORE TEAM. R: A language and environment for statistical computing. Vienna: R Foundation for Statistical Computing, 2014.

RESENDE, M. D. V. Software SELEGEN-REML/BLUP: sistema estatístico e seleção genética computadorizada via modelos lineares mistos. Colombo: Embrapa Florestas, 2007. 359 p.

RESENDE, M. D. V. Genética biométrica e estatística no melhoramento de plantas perenes. Brasília: EMBRAPA Informação Tecnológica, 2002. 975 p

RESENDE, M. D. V. Delineamento de experimentos de seleção para maximização da acurácia seletiva e do progresso genético. Revista Árvore, Viçosa, v. 19, n. 4, p. 479-500, 1995.

RESENDE, M. D. V.; BARBOSA, M. H. P. Melhoramento genético de plantas de propagação assexuada. Colombo: Embrapa Florestas, 2005. 130 p.

SANTOS, G. A.; RESENDE, M. D. V.; SILVA, L. D.; HIGA, A.; ASSIS, T. F. Interação genótipos x ambientes para produtividade de clones de Eucalyptus L'Hér. no estado do Rio Grande do Sul. Revista Árvore, Viçosa, v.39, n.1, p. 81-91, 2015.

SANTOS, G. A.; XAVIER, A.; LEITE, H. G. Desempenho silvicultural de clones de Eucalyptus grandis em relação às árvores matrizes. Revista Árvore, Viçosa, v. 30, n. 5, p. 737-747, 2006.

SEBBENN, A. M.; BÔAS, O. V.; MAX, J. C. M. Variação genética, herdabilidades e ganhos na seleção para caracteres de crescimento em teste de progênies de Pinus caribaea var. bahamensis aos 20 anos de idade em Assis/SP. Revista do Instituto Florestal, São Paulo, v. 20, n. 02, p.103-115, 2008.

SURDI, P. G.; BORTOLETTO JÚNIOR, G.; MENDES, R. F.; ALMEIDA, N. F. Use of hybrid Pinus elliottii var. elliottii x Pinus caribaea var. hondurensis and Pinus taeda L. in the production of OSB panels. Scientia Forestalis, Piracicaba, v. 43, n. 108, p. 763-772, 2015.

TAMBARUSSI, E. V.; SEBBENN, A. M.; MORAES, M. L. T.; ZIMBACK, L.; PALOMINO, E. C.; MORI, E. S. Estimative of genetic parameters in progeny test of Pinus caribaea Morelet var. hondurensis Barret \& Golfari by quantitative traits and microsatellite markers. Bragantia, Campinas, v. 69, n. 1, p. 39-47, 2010.

TEIXEIRA, J. E. C.; BONINE, C. A. V.; DIAS, D. C.; SCARPINATIM E. A.; AGUIAR, A. M.; TOLEDO, F. H. R. B.; TAMBARUSSI, E. V.; VENCOSKY, R. Cruzamentos dialélicos entre clones elite de Eucalyptus grandis e Eucalyptus urophylla. Scientia Forestalis, Piracicaba, v. 41, n. 100, p. 497-505, 2013.

VENCOVSKY, R.; BARRIGA, P. Genética biométrica no fitomelhoramento. Ribeirão Preto: Sociedade Brasileira de Genética, 1992. 496 p.

XIANG, B.; LI, B.; ISIK, F. Time Trend of Genetic Parameters in Growth Traits of Pinus taeda L. Silvae Genetica, v. 52, n. 3-4, p.114-120, 2003.

ZOBEL, B.; TALBERT, J. Applied forest tree improvement. New York: John Wiley \& Sons, 1984. 496 p.

Recebido em 25/09/2017

Aceito em 18/01/2018

Sci. For., Piracicaba, v. 46, n. 119, p. 395-403, set. 2018

DOI: dx.doi.org/10.18671/scifor.v46n119.07 
